\title{
Role of Imaging in Diagnosis, Predicting Biological Activity and in Treatment Plan of Hydatid Disease
}

\section{Mandugula Aruna Devi' ${ }^{1}$, T. Venumadhav², B. Sukanya ${ }^{3}$, Rao T. Manmada ${ }^{4}$, Palanki Gopal5, S. Rammurti ${ }^{1}$}

${ }^{1}$ Radiology, Nizam's Institute of Medical Sciences, Hyderabad, Telangana, India

${ }^{2}$ Surgical Gastroenterology, Nizam's Institute of Medical Sciences, Hyderabad, Telangana, India

${ }^{3}$ Medical Gastroenterology, Nizam's Institute of Medical Sciences, Hyderabad, Telangana, India

${ }^{4}$ Krishna Institute of Medical Sciences, Secunderabad, Telangana, India

${ }^{5}$ Nizams Institute of Medical Sciences, Hyderabad, Telangana, India

Email: arunabilu@yahoo.co.in, thummavenumadhav@yahoo.co.in, sukanyab92@gmail.com, Gopal.polanki@gmail.com,

srammurti@gmail.com

How to cite this paper: Devi, M.A., Venumadhav, T., Sukanya, B., Manmada, R.T., Gopal, P. and Rammurti, S. (2018) Role of Imaging in Diagnosis, Predicting Biological Activity and in Treatment Plan of Hydatid Disease. Open Journal of Internal Medicine, 8, 177-195.

https://doi.org/ 10.4236/ojim.2018.83018

Received: September 24, 2017

Accepted: September 8, 2018

Published: September 11, 2018

Copyright $\odot 2018$ by authors and Scientific Research Publishing Inc. This work is licensed under the Creative Commons Attribution International License (CC BY 4.0).

http://creativecommons.org/licenses/by/4.0/

\begin{abstract}
Cystic Echinococcosis (CE) is a zoonotic parasitic disease caused by the larval stage of the cestode Echinococcus granulosus. Hydatid cyst is a significant universal medical problem. In human cestode involves any organ and develops to hydatid cyst. Liver is the first and most frequently involved organ as the liver is the primary filter station of portal circulation. Though hydatid disease is benign disease, traditionally surgery is the treatment of choice for complete elimination of infective focus, which is associated with considerable mortality, morbidity. Systemic chemotherapy and percutaneous drainage have evolved in the recent past as an alternative to conventional surgery. The safety and success rate of these methods is influenced by the size, stage, location of the cysts and associated complications. Benzimidazoles can be considered in multiple, small hydatid cysts, but large cysts (cysts $>6 \mathrm{~cm}$ ), bone marrow depression, pregnancy are contraindications of pharmacotherapy. Percutaneous drainage combined with ALB is a safe and effective treatment in liver hydatid patients with a surgical contraindication and younger cysts, but the presence of certain radiological signs (pericystic ducts and exophytic components) is a contraindication to non-surgical management because of the danger of biliary obstruction. However, the surgical treatment technique also cannot be standardized, should be tailored according to the cyst relation to the Broncho, biliary, vascular structures and associated complications if any. This review will focus on role of imaging in establishing the diagnosis, in determining cyst location, size, stage of the cyst and in identifying any asso-
\end{abstract}


ciated complications, so that enable the clinician to perform recommended therapeutic procedures for the different cyst types and tailor the procedure performed based on cyst relation to broncho, biliary, vascular structures and associated complications.

\section{Keywords}

USG, CT, MRI, Hydatid Cyst

\section{Introduction}

Human echinococcosis, or hydatid disease is a global zoonotic parasitic disease caused by the larval stage (metacestode) of the parasite that belongs to the family Taeniidae and genus Echinococcus. Four species have been recognized to cause public health concern. Echinococcus granulosus (E. granulosus) is the most common species to cause the human disease known as cystic echinococcosis (CE). Echinococcus multilocularis (E. multilocularis) is rare but most virulent species and causes alveolar echinococcosis (AE). Echinococcus vogelli (E. vogeli) and Echinococcus oligarthrus (E. oligarthrus) cause polycystic echinococcosis. $\mathrm{CE}$ is endemic to countries in the Mediterranean, Turkey, Australia, North Africa, Australia, New Zealand, South America, the Philippines, Northern China and the Indian subcontinent. AE most commonly occurs in China [1] [2] [3] [4].

\section{Discussion}

\subsection{Life Cycle}

Echinococcus has intermediate hosts where immature parasite lives and definitive hosts which harbors the mature parasite. Dogs and Wolves are the definitive hosts. Unlike intermediate hosts, definitive hosts are usually not hurt very much by the infection. The intermediate hosts are grass grazing animals. Humans are accidental secondary hosts [1] because they are usually a dead end for the parasitic infection cycle.

Adult worm resides in the small intestine of the definitive hosts. Worm shreds gravid proglottids that are excreted in the feces. Each proglottid contains hundreds of eggs. Released eggs are ingested by intermediate hosts; Eggs can survive for at least 1yr in the environment. Ingested eggs hatch in the small intestine and release embryo called oncosphere/hexacanth, which penetrates the intestinal mucosa and enters the liver, lungs, or other organs via the blood or lymph, where they mature into hydatid cyst (metacestode larvae) [4] [5] [6]. The life cycle of the tapeworm is completed after the definitive host ingests the organs containing protoscolices (which are produced by germinal layer of $\mathrm{HC}$ ) of the infected intermediate host. Protoscolices evaginate in the intestinal mucosa and develop into adult worms with a scolex, neck and proglottids in 32 - 80 days [2] $[5]$. 


\subsection{Pathogenesis}

Human infection does not occur by the handling or ingestion of meat of infected intermediate host, only occurs via the fecal-oral route, with ingestion of food or water contaminated by the feces of the infected definitive host [5] [7]. Gastric and enteric digestion of eggs facilitates the release of embryos. Primary infestation is the result of the direct evolution of the haxacanth embryo. Embryos subsequently attach to the duodenal or jejunal wall by their hooklets and penetrate the intestinal wall. They reach the liver via the portal circulation. Most of the embryos are stuck in the liver sinusoids, where they either die or grow into hydatid cysts. But embryos with diameters $<0.3 \mathrm{~mm}$ may pass through the hepatic sinusoids and settle in the lungs [2] [3] [4] [7]. Embryos can also reach the lungs via thoracic duct from lymphatics of the small intestine [4] [5] [8], lymphatics of the dome of the liver and the diaphragm, through direct trans diaphragmatic dissemination via broncho, biliary fistula or metastatic. Metastatic lung lesions develop due to rupture of the cyst into right heart chamber, IVC, or rupture of a bone cyst. From the lungs they disseminate to the systemic circulation [9]. Hence the liver is the most frequently involved organ. In humans CE involves the liver in approximately $75 \%$ of cases, the lungs in $15 \%$, and other anatomic locations in $10 \%$ [2] [3] [5] [10]. Isolated involvement of other organs is very rare, involvement of spleen $<2 \%$, kidney $2 \%$ - $3 \%$, adrenal gland $0.06 \%$ to $0.18 \%$, pancreas $0.25 \%$, brain $1 \%-2 \%$, spinal cord $<1 \%$, cardia $0.02 \%-2 \%$ [4] [5] [11] [12]. The growth rate of the hydatid cyst depends on the surrounding tissue elasticity. Lung cysts grow faster than the liver cysts as lungs are softer in consistency [7]. HC contains hydatid sand. Pressure inside the cyst is usually around 35 $\mathrm{cm}$, is generally higher than the intraluminal pressure of the bile duct. High intra cystic pressure inside the cyst is an important indicator of its viability. This high pressure is the cause of communication between cysts and the biliary system.

\subsection{Hydatid Cyst Structure and Diagnostic Methods Used}

Hydatid cyst is the cystic space-occupying lesion [2] [3]. Hydatid cysts are only seen with E. granulosus, E. vogeli, and E. Oligarthrus. The lesion from E. multilocularis is called a "larval mass". These are multiple irregular chambers intermixed with the host fibrous reaction and liver parenchyma [5].

The hydatid cyst has three layers: 1) the outer pericyst, a dense fibrous capsule with calcium is formed as result host immune response to the cyst; 2) the middlelaminated membrane, which is acellular; and 3) the inner germinal layer. The middle laminated membrane and the germinal layer together form the true wall of the cyst, also called endocyst, occasionally the laminated membrane alone is referred to as the ectocyst. The germinal layer produces laminated membrane, brood capsules and protoscoleces. A brood capsule is a fluid -filled vesicle-like structure containing protoscoleces [2] [3] [5] [7]. Protoscoleces are the infectious embryonic tapeworms and will grow to the adult stage after ingestion by a 
definitive host. Laminated layer maintains the physical integrity of the hydatid cyst and permits the passage of nutrients but is impervious to bacteria [6]. It also shields the germinal layer from host immune attack [7]. The thickness of the layers depends on the tissue in which the cyst is located. The layers tend to be thick in the liver, less developed in muscle, absent in bone, and brain [6]. Freed scolices together with brood capsules form hydatid sand. Pulmonary hydatid cysts do not undergo calcification [7]. Brain HC rarely show calcium.

\section{Diagnosis}

The disease often starts without symptoms and this may last for years. The symptoms and signs that occur depend on the cyst's location and size. Depending on the location of the cyst in the body, the patient could be asymptomatic even though the cysts have grown to be very large or be symptomatic even if the cysts are absolutely tiny. In symptomatic patients, the symptoms will depend largely on location of the cyst. E.g. Patients with cysts in the lungs will have a cough, shortness of breath and/or pain in the chest. Patients with cysts in the liver will suffer from abdominal pain, tenderness, hepatomegaly with an abdominal mass, jaundice, fever. In addition, if the cysts rupture while in the body, whether during surgical extraction of the cysts or by trauma to the body, the patient would most likely go into anaphylactic shock and suffer from high fever, pruritus (itching), edema (swelling) of the lips and eyelids, dyspnea, stridor and rhinorrhea.

Diagnostic methods used are imaging techniques and serological methods. Imaging is crucial in diagnosis and classification of $\mathrm{CE}$ as clinical symptomatology and signs are nonspecific. Serology is complimentary to radiological imaging due to high false negative and false positive results [3] [13].

\subsection{Imaging Techniques}

Radiography, ultrasonography (US), computed tomography (CT), and magnetic resonance (MR) imaging, MRCP and ERCP are various imaging modalities. The imaging method used depends on the organ involved and the growth stage of the cyst. Imaging appearances depends on age of the cyst and associated complications, and are identical independent of organ involved [1] [3] [5] [14] [15].

Specific signs on imaging are visualization of calcification of the cyst wall, presence of the daughter cysts, and membrane detachment [Figures 1-4]. The diagnosis of $\mathrm{HC}$ at early stage is difficult [3] [4] [5].

USG is preliminary and cardinal imaging modality for diagnosis, classification and follow up of patients with liver HC; As USG has highest sensitivity for the detection of hydatid sand in purely cystic lesions, floating membranes, daughter cysts and vesicles [Figure 5]. WHO informal working group on Echinococcus (IWGE) classification of liver CE, which is originally developed by Gharbi and colleagues in 1981, which is based on USG imaging appearances of the cyst which vary depending on the stage of the cyst, is the currently widely used 


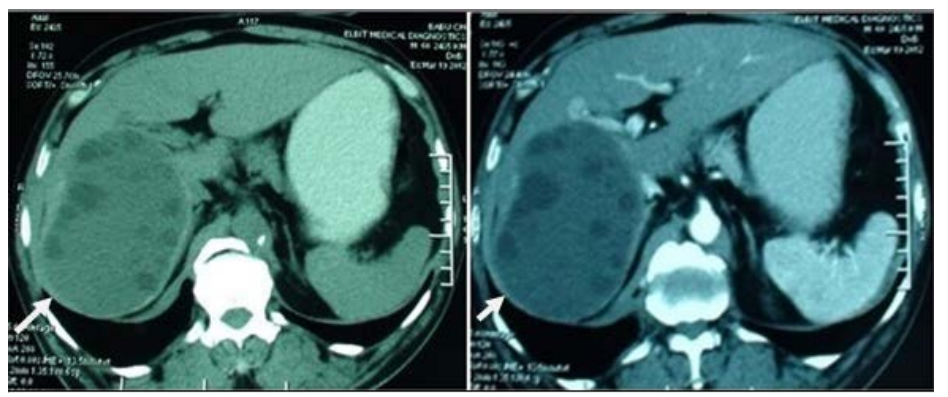

Figure 1. Class CE2 Hydatid cyst in liver in a 60 yr male patient with history of right hypochondriac pain. Plain and CECT abdomen showed multivesicular cystic lesion with attenuation of mother cyst is higher than that of daughter cysts and thin high attenuation peripheral rim on plain CT with no enhancement on postcontrast.

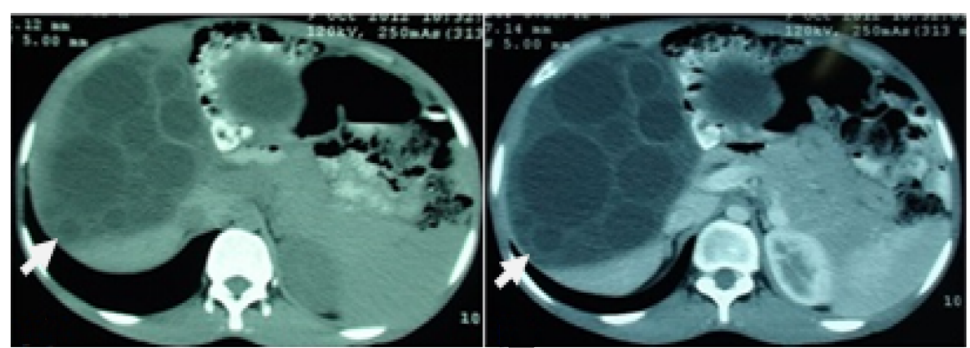

(a)

(b)

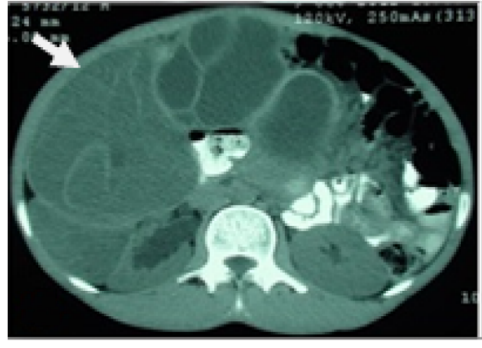

(c)

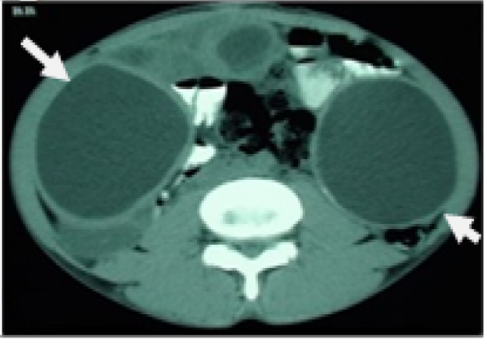

(d)

Figure 2. Multiple intraperitoneal Hydatid cysts in mesentery and liver in a $22 \mathrm{yr}$ male patient with right hypochondriac and epigastric pain with early satity. Plain and CECT abdomen showed Class CE2 cyst in liver, Class CE1 and CE3 cysts in mesentery. Most of the cysts show hyperdense peripheral rim on plain CT. Class CE3 cyst is deformed with floating membranes within.

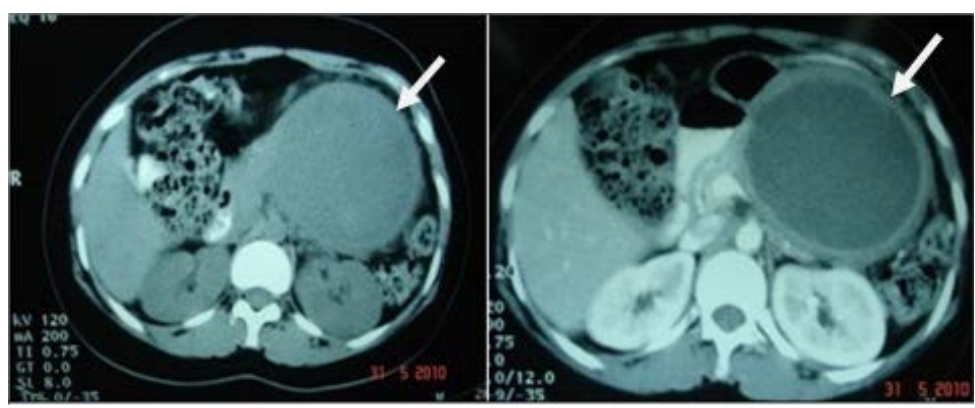

Figure 3. Class CE1 Hydatid cyst in pancreas in a $16 \mathrm{yr}$ old female patient with history of abdominal discomfort. Plain and CECT abdomen showed rounded, unilocular cystic lesion with thin high attenuation rim with no enhancement on postcontrast involving body and tail of pancreas. 


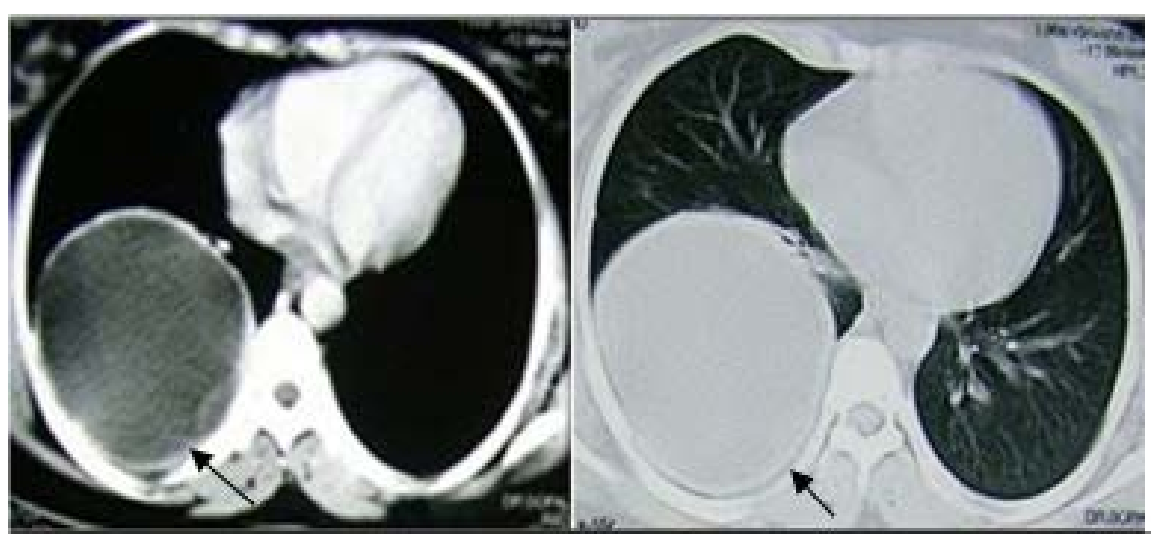

Figure 4. Class CE2 hydatid cyst in right lung in a $35 \mathrm{yr}$ female patient with history of cough, expectoration and fever. Plain and CECT chest revealed rounded, thin walled, unilocular cystic lesion with daughter cyst within, with no enhancement on postcontrast.

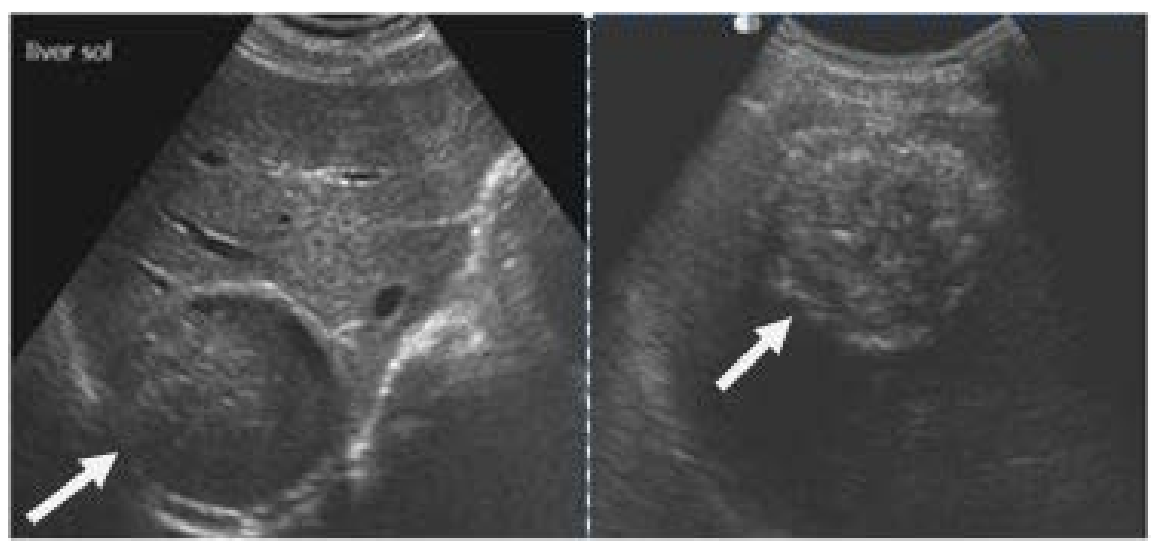

Figure 5. Class CE1 Hydatid cyst in liver in a $53 \mathrm{yr}$ old gentleman came for routine health check up. USG abdomen showed thin walled, unilocular cyst with falling snow flakes sign "hydatid sand".

system for staging CE so as to enable the clinician to perform recommended therapeutic procedures for the different cyst types. [2] [3] [5] [10] [13] [16].

Radiograph is primary imaging modality of choice in case of $\mathrm{HC}$ bone and lung [5]. Uncomplicated pulmonary HC appear as sharply defined, round-to-oval, homogenous opacity [Figure 6]. Pulmonary hydatid cysts do not undergo calcification and daughter cyst formation is also rare, so difficult to differentiate from other lesion. The cyst may become bizarre shaped/polycyclic configuration by the pressure from adjacent broncho-vascular structures, mediastinum, pleura, and adjacent cysts [5] [9]. Multiple large masses in the lungs are pathognomonic for hydatid cysts [7].

CT is the modality of choice in case of HC located in lung, bone, cysts with wall calcifications in other location to demonstrate the internal morphology [Figure 4 and Figures 6-12]. And plays vital role in preoperative evaluation for detection of complications such as biliary, vascular and bronchial involvement, cyst ruptures, and underlying infections [Figure 6, Figure 9, Figures 11-14]. Unruptured cyst is differentiated from other simple cysts by the presence of high 

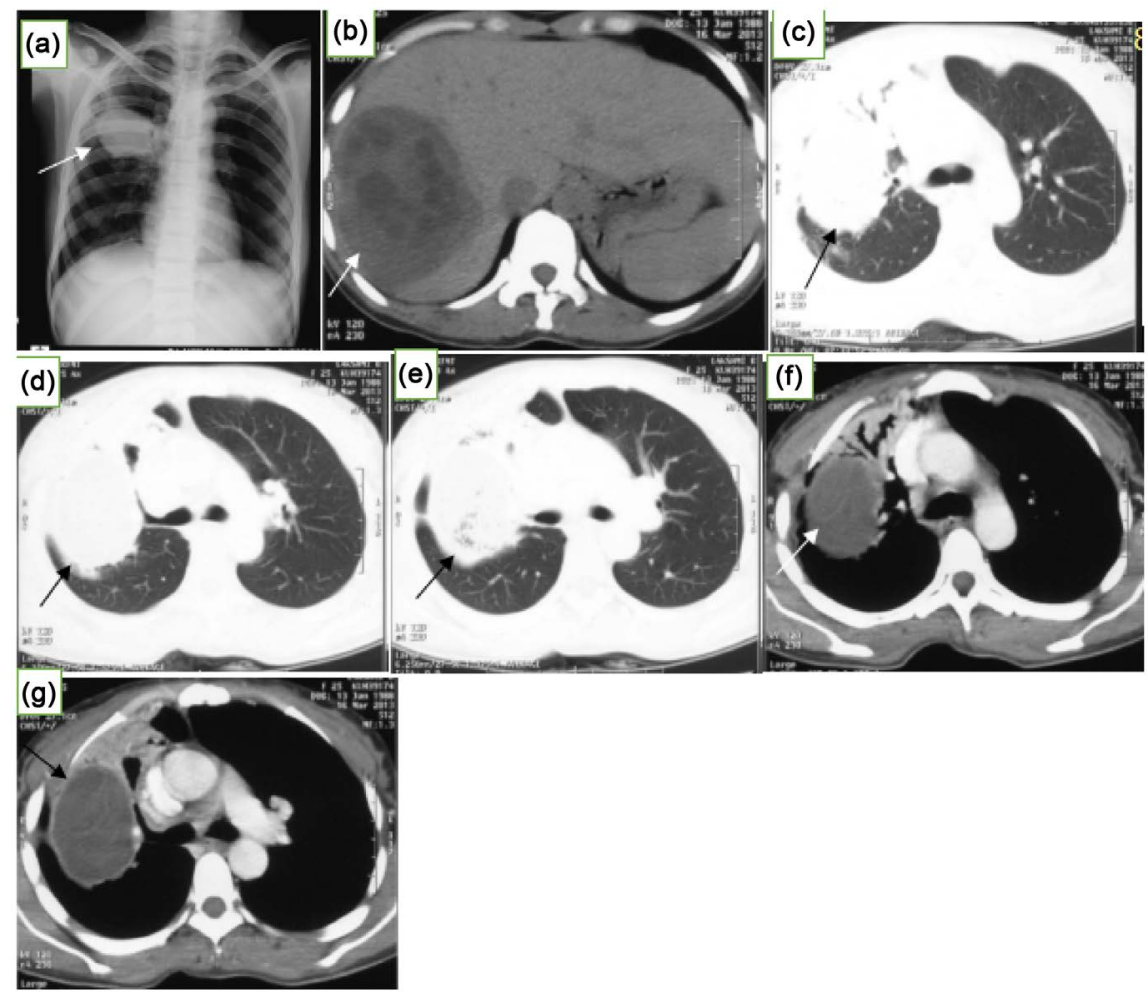

Figure 6. Class CE3 hydatid cyst in upper lobe right lung with associated atelectasis and mild cylindrical bronchiectasis and Class CE2 hydatid cyst in liver in a $25 \mathrm{yr}$ female patient with h/o fever, cough with expectoration. Plain and CECT chest showed well defined, thin walled cystic lesion with "floating membranes within" with no enhancement on postcontrast and adjacent atelectasis with mild cylindrical bronchiectasis. Liver showed thin walled, multivesicular cystic lesion with attenuation of mother cyst is higher than that of daughter cysts with no enhancement on postcontrast.
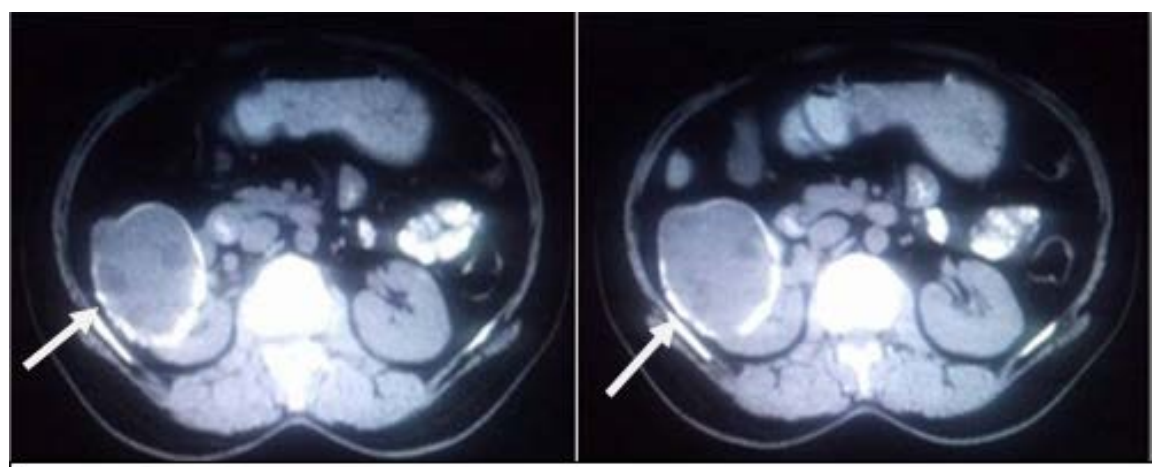

Figure 7. Class CE2 Hydatid cyst in right kidney in a $69 \mathrm{yr}$ old gentleman with history of right loin pain. Plain and CECT abdomen showed well defined, rounded, iso dense lesion, with thick wall calcifications, and few daughter cysts within with differential attenuation, with no enhancement on postcontrast.

attenuation wall at unenhanced CT due to laminated membrane is hyperdense on non-contrast CT; may show indirect signs of infection and/or communication with the biliary tree such as gas/ air fluid levels or fat inside the cysts [Figure 14] [2] [3] [5]. 


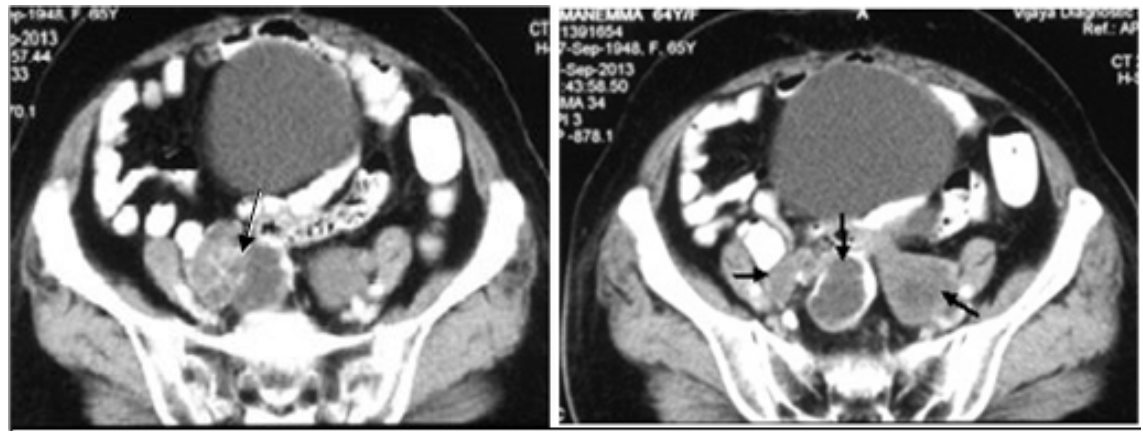

Figure 8. Recurrent hydatidosis pelvis in a $64 \mathrm{yr}$ female patient with history of salphingo-oophorectomy for ovarian hydatid cysts. CECT abdomen showed multiple cysts at various stages. Class CE1 cyst is iso dense with peripheral high attenuation rim, class CE2 cyst filled with multiple vesicular cysts with "spoke wheel" appearance with high attenuation wall and internal septa, another CE5 cyst with thick peripheral calcifications.

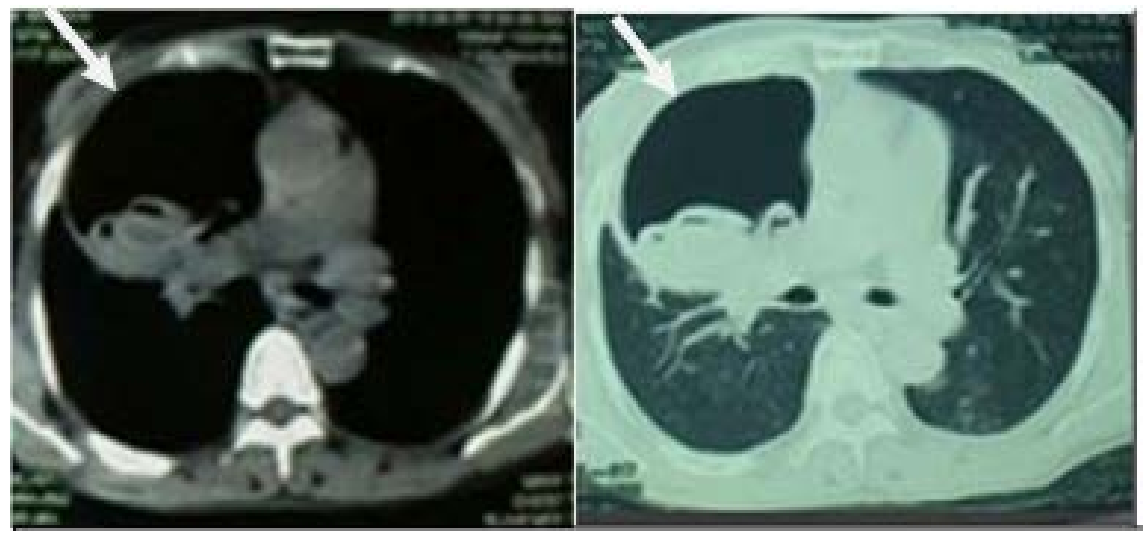

Figure 9. Complicated class CE3 hydatid cyst in right lung in a $69 \mathrm{yr}$ old female patient with history of cough with expectoration, Plain CT chest revealed thin walled, air filled cavity with twisted membranes within, with "spin/whirl" appearance.
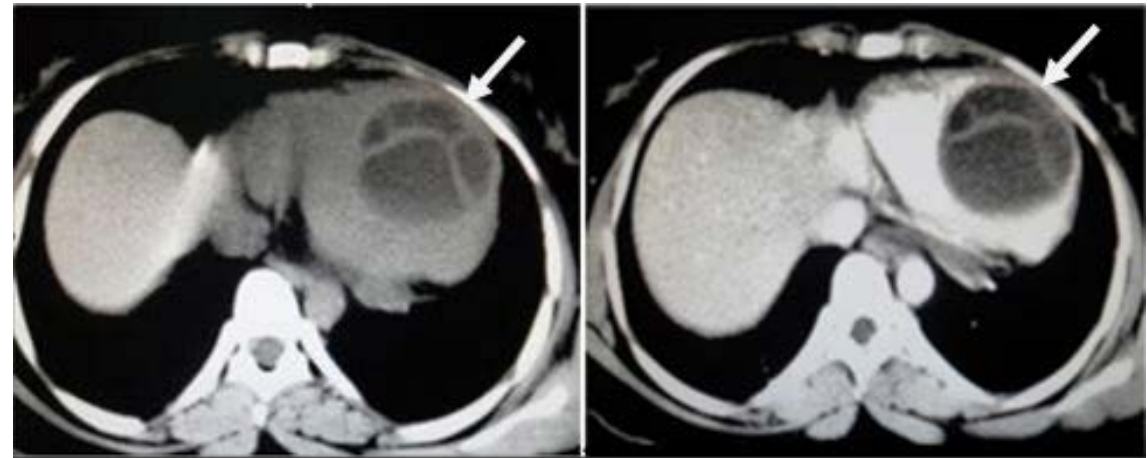

Figure 10. Class CE2 hydatid cyst in myocardium of ventricle in a $25 \mathrm{yr}$ female patient with history of chest pain. Pain and CECT chest showed well defined, thin walled cystic lesion with internal loculations with "rosette-like appearance" with no enhancement on post contrast in myocardium of ventricle.

MR is the indispensable in imaging HCs of the central nervous system as the brain HC rarely show calcium [2] [3] [5], and in preoperative evaluation for detection of complications such as biliary, vascular and bronchial involvement, 


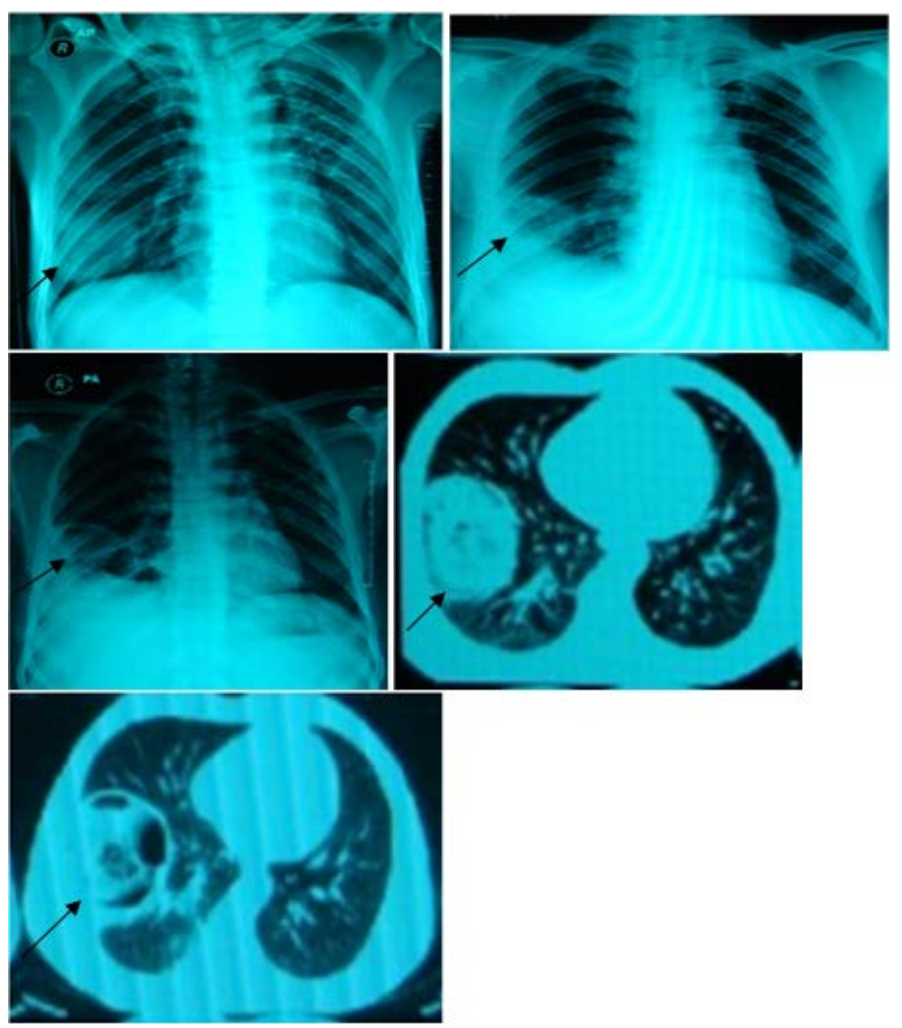

Figure 11. Class CE2 hydatid cyst in right lung in a $34 \mathrm{yr}$ male patient with history of cough with expectoration and fever. Serial plain radiographs chest showed rounded homogenous opacity on initial radiograph followed by "combo sign" with surrounding consolidation, followed by signet ring appearance on subsequent radiographs. Serial plain CT chest showed nonhomogenous, rounded consolidation with "combo sign" due to air pockets in endocyst due to communicating rupture into bronchus followed by round consolidation with "signet ring" appearance due to air bubbles between pericyst and endocyst on subsequent $\mathrm{CT}$ due to impending rupture.

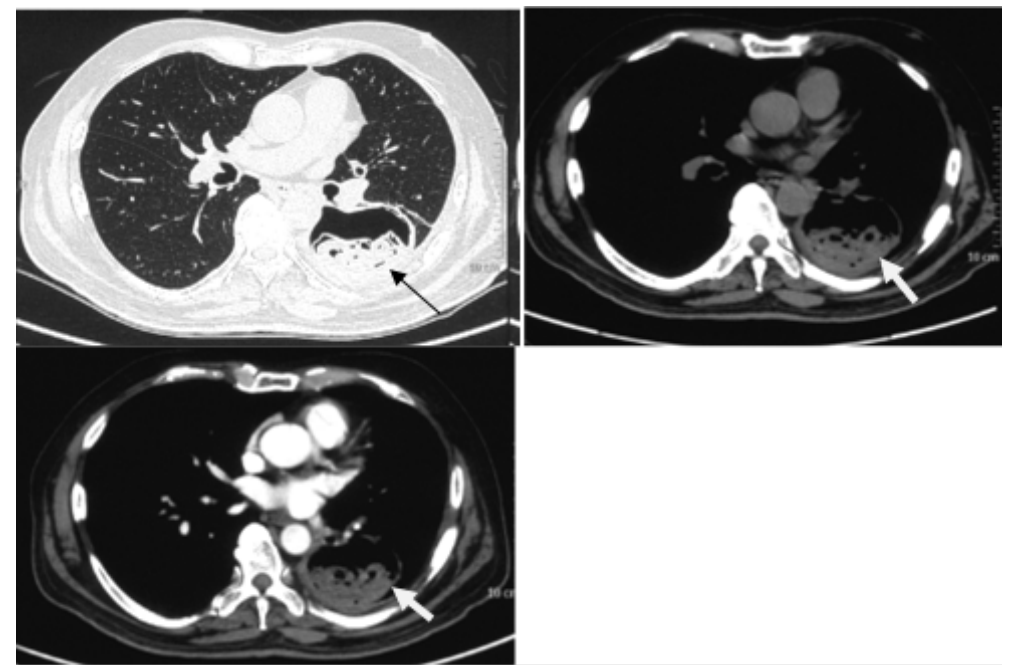

Figure 12. Class CE3 hydatid cyst in left lung in a $59 \mathrm{yr}$ male patient with h/o cough with expectoration. Plain and CECT chest showed thin walled cavitary lesion with "rising sun" appearance due to confluent rounded densities (daughter cysts) in the bottom of the cyst with wavy interface with no enhancement on postcontrast. 


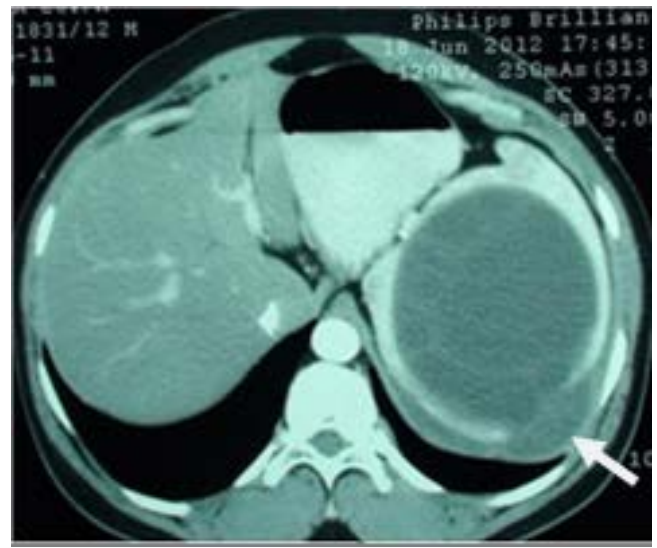

Figure 13. Class CE2 Hydatid cyst in spleen in a 28 yr male patient with left hypochondriac pain, non radiating. CECT abdomen showed thin walled multi locular cyst with "rossatte-like" appearance with exophytic component adherent to left dome of diaphragm.
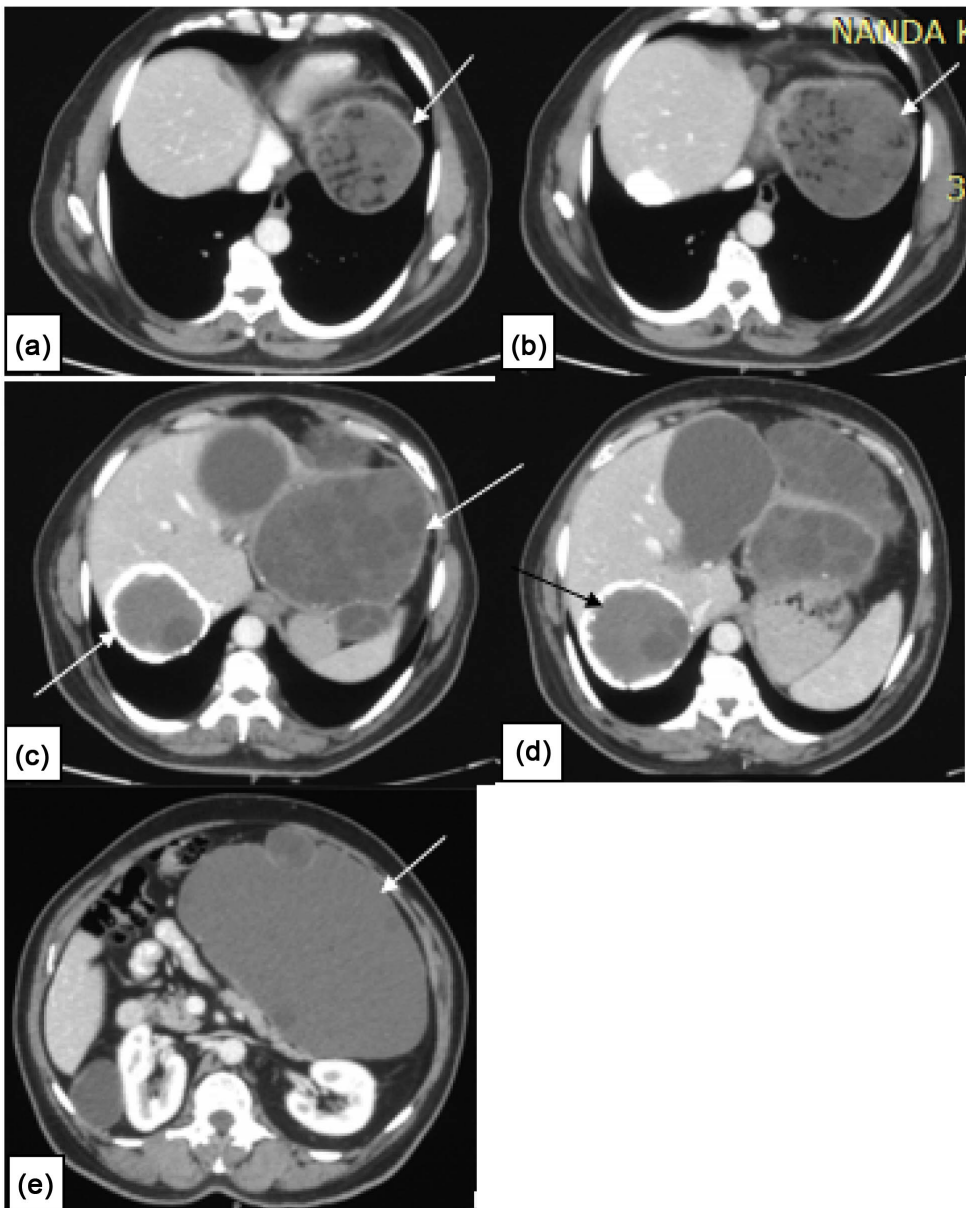

Figure 14. Multiple Hydatid cysts at various stages and one with complication in liver in a $59 \mathrm{yr}$ male patient with epigastric pain with early satity and recent onset jandice. CECT abdomen showed Class CE5 cyst in right lobe, Class CE1 and CE2 cysts in left lobe. Cyst in the right lobe showed thick rind of peripheral calcium with few daughter cysts within. CE2 cyst in the left lobe showed large exophytic component and fat attenuation areas within suggestive of biliary communication. 
cyst ruptures. MRI is as sensitive as USG for the detection of hydatid sand in purely cystic lesions, floating membranes, daughter cysts and vesicles. The cyst is hyperintense on $\mathrm{T} 2 \mathrm{~W}$ images and is surrounded by a low signal rim which is more likely due to collagen rich peri cyst [3] [4] [5] [6]. DW-MRI with high b-factor differentiates simple cyst from CE1 hydatid cysts, hydatid cysts are hyperintense on DW-MRI but simple cyst does not show significant hyperintensity; ADC of the hydatid cyst is lower than that of simple cyst $\left(2.5 \times 10^{-3} \pm 0.9 \mathrm{vs}\right.$. $3.5 \times 10^{-3} \pm 0.5$ ) [3] [5] due to viscous hydatid cyst contents.

ERCP and MRCP demonstrate cystobiliary relationship, dilated bile ducts and daughter vesicles or germinative membranes of cysts in bileducts. But are not effective in demonstrating communication of cyst with bileducts due to high intracystic pressure [Figure 15] [1] [5].

\subsection{WHO-IWGE Classification of Liver CE and Appearances of Each with Various Imaging Modalities}

WHO-IWGE classification of Ultrasound images of cystic echinococcosis cysts

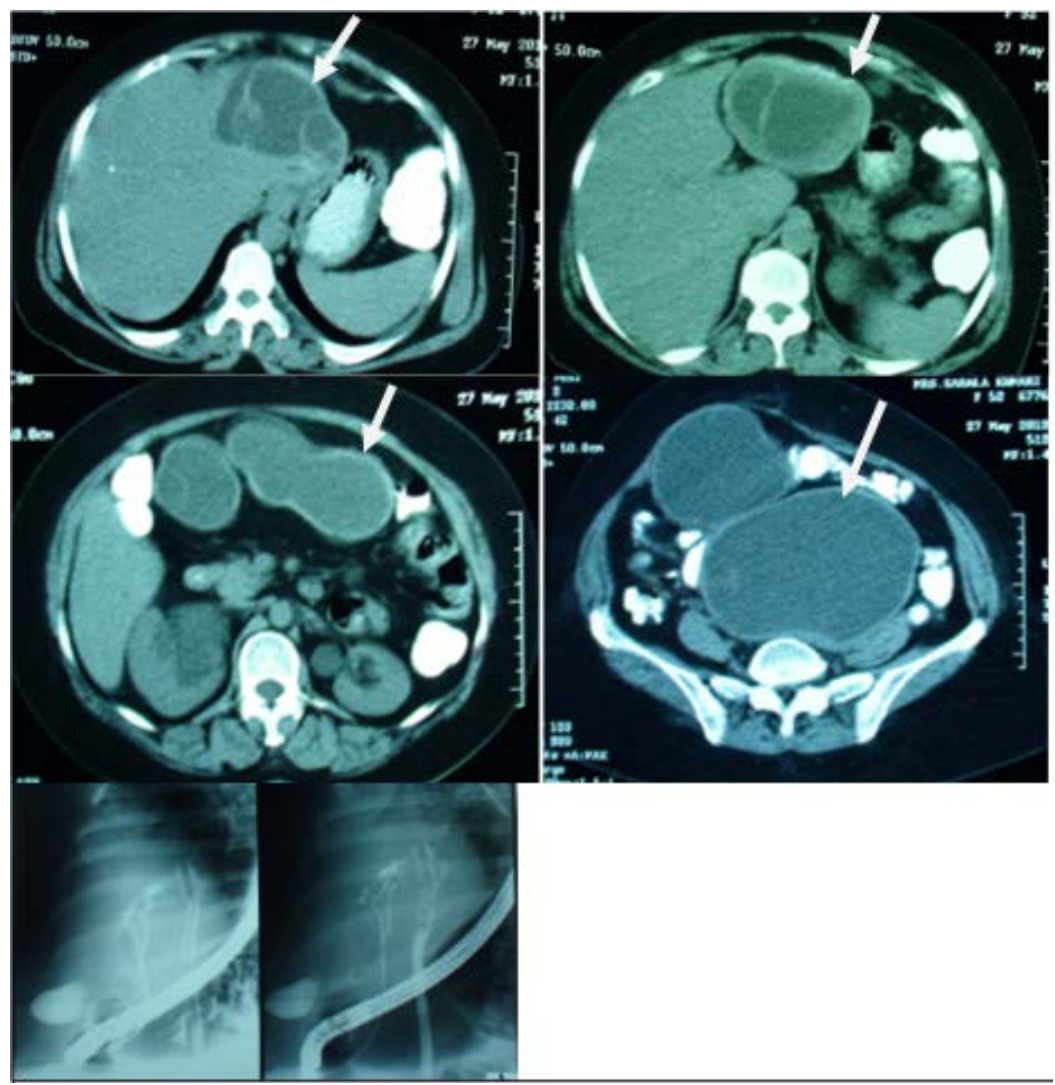

Figure 15. Large Class CE2 hydatid cyst in left lobe of liver with exophytic component and Multiple class CE1 and CE2 cysts in the peritoneal cavity in a $52 \mathrm{yr}$ female patient with history of distension and pain abdomen. CECT abdomen showed large cystic lesion with multiple internal loculations and septa in left lobe liver with exophytic component; multiple other thin walled, unilocular and multilocular cystic lesions in the peritoneal cavity and in pelvis. Post -operative ERCP showed suggestion of biliary communication of left lobe lesion. 
based on morphologic characteristics in association with biological activity to predict the clinical fate of the cyst and enable clinician to perform recommended therapeutic procedures for the different cyst types [17] [18] [19]. MRI reproduces the ultrasound-defined features of CE better than CT, since the highly specific cyst stage defining features are features of the cyst matrix with the exception of the double line sign of CE1, where a cyst wall feature is diagnostic. Thus MRI with heavily $\mathrm{T} 2$ weighted series is preferable to CT if US cannot be performed due to cyst location or patient-specific reasons [13].

CL: Unilocular, cystic lesion(CL) with uniform anechoic content, not clearly delimited by hyperechoic rim (cyst wall); normally round but may be oval with variable size, usually small (CLs) $<5 \mathrm{~cm}, \mathrm{CL} \mathrm{m} 5-10 \mathrm{~cm}$, CL l $>10 \mathrm{~cm}$. If CE active and cystic lesions are caused by $\mathrm{CE}$ at an early stage of development then usually these cysts are not fertile. USG does not detect any pathognomonic signs. These cysts need further diagnostic work up.

CE1: Usually fertile.

USG: Unilocular, simple cyst, round or oval, with visible wall, with or without hydatid sand and septa. Rolling the patient during evaluation disperses the sand, creating small echogenic foci, or "falling snow flakes" [Figure 5].

CT: Appears as well defined, water-attenuation mass. Frequently, the septa and cyst wall enhance after IV contrast administration.

MRI: Lesion appears hypointense on T1W and hyperintense with hypointense rim on $\mathrm{T} 2 \mathrm{~W}$

CE2: usually fertile.

USG: Multi vesicular, multiseptated cysts with visible wall; Cyst septations produce "wheel-like" structures, and presence of daughter cysts is indicated by "rosette-like" or "honeycomb-like" structures. Daughter cysts may partly or completely fill the unilocular mother cyst.

CT: Type II HCs are sub classified into 3 types based on the age, number, and arrangement of the daughter cysts. Type IIA lesions contain round daughter cysts arranged at the periphery. The average $\mathrm{CT}$ attenuation of the mother cyst is higher than that of daughter cysts [Figure 1].

Type IIB lesions contain larger, irregularly shaped daughter cysts occupy the almost entire volume of mother cyst. The high attenuation fluid within the mother cyst that surround the daughter cysts looks like septa, creating a "rosette appearance" [Figure 2(a) and Figure 2(b), Figure 10, Figure 13, Figure 16].

Type IIC lesions appear as relatively high attenuation round or oval masses with scattered calcifications and occasional daughter cysts. These findings represent degeneration of old cysts with amorphous and tenacious content [Figure 7 and Figure 8].

MRI: Daughter cysts may appear hypointense or isointense relative to the maternal matrix on T1W and $\mathrm{T} 2 \mathrm{~W}$ images.

CE3: transitional stage, which may degenerate further or may give rise to daughter cysts [Figure 2, Figure 9, Figure 12]. 

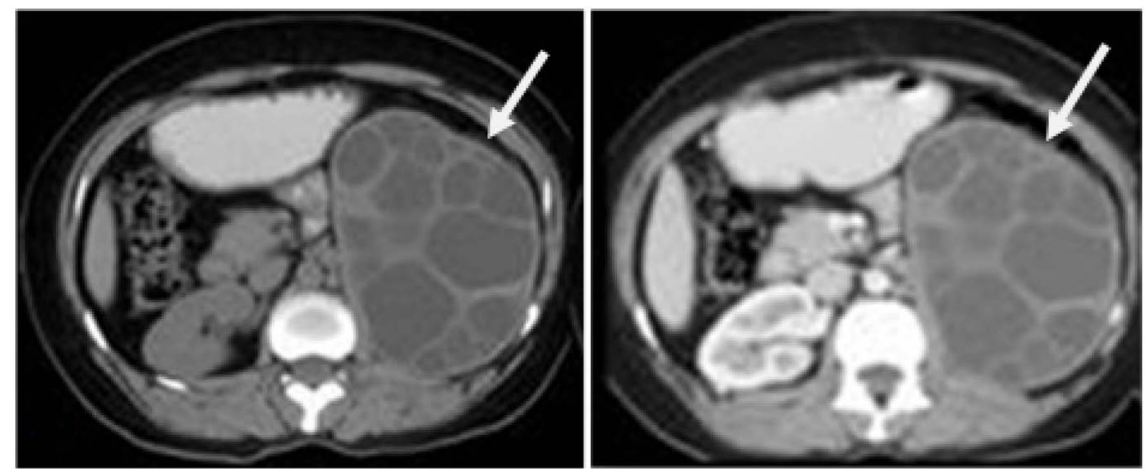

Figure 16. Class CE2 Hydatid cyst in left kidney in a $32 \mathrm{yr}$ male patient with left flank pain. Plain and CECT abdomen showed oval shaped, multivesicular cystic lesion with thin high attenuation rim on plain CT with no obvious enhancement on postcontrast with "Honey comb-like" appearance in left kidney.

USG: Unilocular cyst with anechoic content, with detachment of laminated membrane from the cyst wall visible as floating membrane or as "water-lily sign" which is indicative of wavy membranes floating on top of remaining cyst fluid, cyst form may be less rounded because of decrease of intra cystic fluid pressure, which may contain daughter cysts/cyst with daughter cysts in solid matrix.

MRI: Daughter cysts may appear hypointense or isointense relative to the maternal matrix on T1W and T2W images. Detached, floating membranes within the cyst which create "serpent sign" or "snake sign" appear hypointense with all sequences.

CE4: Are inactive, most of these are not fertile.

USG: Heterogenous hypoechoic or hyperechoic degenerative contents with no daughter cysts. May show a "ball of wool" sign which is indicative of degenerating membranes. US features are not pathognomonic and further diagnostic work up required to ascertain diagnosis.

CE5: Are inactive, non fertile in most cases.

USG: Cyst characterized by thick calcified wall that is arch shaped, producing a cone shaped shadow. Degree of calcification varies from partial to complete. Diagnosis is uncertain. Features are not pathognomonic but highly suggestive for E. granulosus.

CT and MRI: Dead cysts with partial or total calcifications. Calcified or hyperattenuating on CT, hypointense on MRI.

Complicated cysts: Complications include rupture and superinfection and may be seen in type I and type II. Cyst rupture is mainly due to degeneration of parasitic membranes, risk of rupture increases with the size, anthelminthic therapy and percutaneous aspiration [7]. Cyst rupture may be contained, communicating, or direct. In contained rupture, the endocyst ruptures and the cyst contents are confined within the pericyst [Figure 2, Figure 9, Figure 12]. In communicating rupture, the cyst ruptures into the biliary and bronchial radicles lying in the pericyst, rarely into vascular system, such as portal vein, IVC, pulmonary artery, rare but cyst rupture into IVC and right sided cardiac chambers 
present as recurrent cystic pulmonary embolism. They may or may not be infected [5]. Indirect signs of communicating rupture are deformity of cyst, defect in the wall, presence of beak like projection extending from cyst wall, dilated bile ducts and daughter vesicles or germinative membranes of cysts in bile ducts on MRCP/ERCP, presence of fat inside the cysts on CT/MRI in cysts with biliary tree communication [Figure 14]. Direct sign is visible communication but is difficult to demonstrate with imaging due to high intra cystic pressure than bile ducts. [2] [3] [5] [6] [7] [8]. Rupture into the bronchus is the most frequent complication of pulmonary hydatid disease, produces an allergic pneumonitis in the surrounding areas. This is reversible up to 10days and after 10 - 14 days secondary bacterial pneumonia develops. In direct rupture, both the endocyst and the pericyst rupture, with spillage of cyst contents into the peritoneal, pleural cavities or other structures, rarely into a hallow viscus, such as stomach or colon, into ventricular system, subarachnoid spaces, pcs. Direct rupture has the greatest clinical consequences including anaphylaxis, hydatid dissemination, secondary bacterial infection, and recurrences after surgery. Peripherally located cysts are more prone to direct rupture due to deficient pericyst and little host tissue support [3] [5] [7]. Indirect signs of infection are gas/air fluid levels [Figure 9, Figure 11, Figure 12].

\subsection{Various Imaging Signs of Hc}

1) Daughter cysts 2) Hydatid membranes and 3) Hydatids and are the signs seen in the early stages, which change their position with change in position of the patient [3]. 1) Snake/serpent sign (detached, undulating membranes. 2) Spin/Whirl sign (in advanced stage of collapse, the membranes appear twisted on imaging). 3) Water-Lily sign (collapsed endocyst layer floating on a layer of fluid within the cyst). 4) Double-line sign (axial sonogram delineates the space between parasite's ectocyst and host's pericyst) are the signs seen in the later stages when the dissection takes place. 5) In case of E. multilocularis infection USG images show the typical Hailstorm pattern (characterized by multiple echogenic nodules with irregular and indistinct margins) [3] [4]. 7) Notch sign with bilobed appearance of pulmonary $\mathrm{HC}$ due to indentation produced by adjacent relatively rigid anatomical structures. 8) The shape of the cyst may change radiologically during maximal inspiration and expiration known as the Escudero-Nimerov-sign [5] [7]. 9) Slot sign due to loss of sperical shape with small depression resulting in reniform shape which may suggest bronchial rupture. Bronchial communication of the pulmonary cyst produces various radiographic appearances; 1) Crescent sign or meniscus sign due to air between pericyst and laminated membrane, 2) Air fluid level and cumbo sign/double arc sign due to air entry into endocyst and radiolucent rim between the pericyst and endocyst, 3) Water-lily sign/camolette sign due to collapsed membranes floating on cyst fluid, 4) Rising sun sign due to rounded radio opacities of daughter cysts at the bottom of the cyst due to rupture of endocyst, 5) Signet ring sign due to blebs of air between pericyst and en- 
docyst suggest impending rupture, 6) Air-buble sign due to presence of air bubbles in regions surrounding the cyst due to cyst rupture secondary to bacterial infection [5] [6] [7].

The more complex cysts may mimic solid hepatic masses, Presence of membranes and/or daughter cysts rules out other cystic lesions. In case of multi vesicular hydatid cyst: peripheral daughter cysts attached to the membrane of the mother cyst in the center and difference of density (density of the mother cyst is higher than that of the daughter cyst because of advanced degenerative changes) are characteristic CT findings. After medical therapy, detached membranes within the degenerated cyst indicate that parasite is responding to medical therapy [3] [4] [7].

\subsection{Serology Testing}

Serological tests complimentary to imaging diagnosis [12] [20]. The majority of the serological tests are based on detecting antibody as it is more sensitive than antigen detection. The enzyme-linked immunosorbent assay (ELISA), the indirect hemagglutination antibody assay (IHT), latex agglutination test, and immunoblot test (IB) are commonly used serological methods. Specific confirmation tests of reactivity are immune diffusion, immunofluorescence antibody test and arc-5 immune electrophoresis (IEP). Most commonly used antigens are antigen 5 and antigen B [4] [10] [18] [21]. However, serological tests have certain disadvantages. Sensitivity rates of these immunodiagnostic tests vary from $60 \%$ to $90 \%$. Because seropositivity depends on the physical location, integrity, and vitality of the larval cyst and the assay format; tests are least sensitive in patients with intact hyaline cysts, usually negative in patients with calcified or dead cysts [14] [16] [18] [22]. 1. Cysts in the lungs, brain, and spleen are associated with lowered serodiagnostic immune reactivity [22]. They may cause a false-positive reaction in people with other helminthic infections for example E. multilocularis and Taenia solium due to cross reactions. Other causes include patients with cancer, chronic immune disorders, cirrhosis of liver, and presence of anti-PL antibodies [4]. A secondary test is performed using either an Arc5 test, IgG4-ELISA, or immunoblot for antibodies against E. granulosus antigens to rule out false positive cross-reactivities. Another limitation of the serological tests is in monitoring patients after surgery or pharmacotherapy as the antibody titer rises at 1 3 months after surgery and starts receding after 3 months. It takes another 12 24 months to become negative [16]. The sensitivity is higher in patients with multiorgan involvement $(90 \%-100 \%)$, and in the case of ruptured cysts. Serological methods can predict the either progression or regression of the cyst based on IgG4 and IGE antibodies [2] [4].

\section{Treatment}

Treatment depends on location, size, age of the cyst, and complications of the cysts. Chemotherapy is palliative as it is poorly absorbed by the intestine and is 
unable to diffuse across the cyst wall [4] [14] [18]. However chemotherapy is valuable in smaller CE1 pulmonary cysts, multiple small cysts, multi organ disease, patients with contraindication for surgery, refusal for surgery, recurrent cysts, and patients with intraoperative spillage of hydatid fluid [2] [4] [14] [18]. Contraindications of pharmacotherapy are large cysts $(>6 \mathrm{~cm}$ diameter) that are at risk of rupture and superficial cysts, inactive or calcified cysts, cyst with multiple internal septations, infected cysts, chronic liver disease, bone marrow depression, pregnancy [1] [18] [23].

Larger CE1 cysts up to $10 \mathrm{~cm}$ diameter are ideal for PAIR. CE1 and CE3a type cysts can be treated by percutaneous methods + Chemotherapy. Contraindication to non-surgical management are presence of pericystic ducts, exophytic components because of the danger of biliary obstruction and risk of spillage, cysts in lung, spine, brain and heart, cysts communicating with the biliary tree, bronchi, urinary tract, noncooperative patients and inaccessible or risky location of the cysts [2] [11] [17] [18] [24] [25]. PAIR in conjunction with ALB/MBZ is more effective for cysts $<5 \mathrm{~cm}$. For cysts measuring $>5 \mathrm{~cm}$ PAIRD is preferable. If numerous and large daughter cysts are present PPDC (percutaneous puncture with drainage and curettage) is preferable [2] [14] [17]. But there is not sufficient evidence to support its use as a standard procedure for patients with uncomplicated cysts, so the use of PAIR as treatment must be discussed on a case by case basis whatever the setting and/or the country [11].

Response to PAIR is monitored with sonography. Reduction in the size of the cavity, membrane detachment, wall calcification, increased areas of solidification in the cyst, and increased echogenicity of the cyst (consistent with pseudo mass appearance) are indicators of good response to treatment [2] [3] [24] [26].

Advanced stage CE4 and CE5 can be left alone (watch and wait). CE2 and the transitional cyst stage CE3b often need Surgery+ Chemotherapy/percutaneous intervention (non-PAIR) for definite cure [1] [2] [13]. Surgical treatment technique cannot be standardized, and surgical technique should be tailored according to the extent of the cyst and any associated complications of the cyst such as opening into the bile ducts or peritoneal cavity. Thus identification of cystobiliary or cystobronchial communication is critical [1] [13].

Radical surgery for liver HC refers to pericystectomy and liver resection, are curative with lower recurrence rates and is the preferred method in patients with superficial and exophytic hydatid cysts. But radical surgery is associated with significant mortality and morbidity for such a benign disease [2].

Conservative surgery refers to removal of the cyst content and sterilization of the residual cavity, together with partial cyst resection, conservative procedures with omentoplasty is the safe, simple, effective; Is the preferred method for cysts located centrally, lying close to major biliovascular channels and around the hilum [1] [18] [25].

External tube drainage is recommended for infected cysts and common bile duct exploration + a biliary drainage procedure must be added to external tube 
drainage for cysts with intrabiliary rupture. Treatment must be discussed on a case by case basis whatever the setting and/or the country.

Prevention of cystic disease is by treating dogs that may carry the disease and vaccination of sheep [27]. Treatment is often difficult. The cystic disease may be drained through the skin, followed by medication. Sometimes this type of disease is just watched. The alveolar type often needs surgery followed by medications. The medication used is albendazole, which may be needed for years. The alveolar disease may result in death. It is necessary to prevent the infection in sheeps as they are the main source of meat in many countries in the world and are significant reservoir to canine and human infection. The EG95 vaccine made of PSTSA has proven to be highly effective. Studies showed that two or three times of immunization with the PSTSA is an effective and immunogenic compound for immunization of sheep against CE. But the lasting time of protection in created immunity has not yet been determined [28] [29].

\section{Conclusion}

Imaging has pivotal role in establishing the diagnosis of hydatid cyst and to guide management decisions based on cyst class and associated complications if any; as percutaneous drainage combined with ALB is a safe and effective treatment in liver hydatid patients with a surgical contraindication and younger cysts, but the presence of certain radiological signs (pericystic ducts and exophytic components) is a contraindication to non-surgical management because of the danger of biliary obstruction. However, the surgical treatment technique also cannot be standardized and should be tailored according to the cyst relation to the Broncho, biliary, vascular structures and associated complications if any.

\section{Conflicts of Interest}

The authors declare no conflicts of interest regarding the publication of this paper.

\section{References}

[1] Ezer, A., Nursal, T.Z., Moray, G., Yildirim, S., Karakayali, F., Noyan, T. and Haberal, M. (2006) Surgical Treatment of Liver Hydatid Cysts. HPB (Oxford), 8, 38-42. https://doi.org/10.1080/13651820500468000

[2] Pakala, T., Molina, M. and George, Y. (2016) Hepatic Echinococcal Cysts: A Review. Journal of Clinical and Transplantational Hepatology, 4, 39-46. https://doi.org/10.14218/JCTH.2015.00036

[3] Marrone, G., Crino, F., Caruso, S., Mamone, G., Carollo, V., Milazzo, M., Gruttadauria, S., Luca, A. and Gridelli, B. (2012) Multidisciplinary Imaging of Liver Hydatidosis. World Journal of Gastroenterology, 18, 1438-1447. https://doi.org/10.3748/wjg.v18.i13.1438

[4] Rasheed, K., Zarqar, S.A. and Telwani, A.A. (2013) Hydatid Cyst of Spleen: A Diagnostic Challenge. North American Journal of Medical Sciences, 5, 10-20. https://doi.org/10.4103/1947-2714.106184

[5] Mehta, P., Prakash, M. and Khandelwal, N. (2016) Radiological Manifestations of 
Hydatid Disease and Its Complications. Journal of Tropical Parasitology, 6, 103-112. https://doi.org/10.4103/2229-5070.190812

[6] Malik, A., Chandra, R., Prasad, R., Khanna, G. and Thukral, B.B. (2016) Imaging Appearances of Atypical Hydatid Cysts. Indian Journal of Radiology and Imaging, 26, 33-39. https://doi.org/10.4103/0971-3026.178284

[7] Garg, M.K., Sharma, M., Gulati, A., Gorsi, U., Aggarwal, A.N., Agarwal, R. and Khandelwal, N. (2016) Imaging in Pulmonary Hydatid Cysts. World Journal of Radiology, 8, 581-587. https://doi.org/10.4329/wjr.v8.i6.581

[8] Makni, A., Jouini, M., Kacem, M. and Safta, Z.B. (2012) Acute Pancreatitis Due to Pancreatic Hydatid Cyst: A Case Report and Review of the Literature. World Journal of Emergency Surgery, 7, 7. https://doi.org/10.1186/1749-7922-7-7

[9] Akman, R.Y., Sargin, S.Y. and Yazicioglu, A.H. (2000) Hydatid Disease of the Kidney: A Retrospective Review of 13 Cases. Infections in Urology, 13.

[10] Lt Col, A.S., Brig, S.R. and Sqn Ldr, R.M. (2012) Management of Liver Hydatid Cysts-Current Perspectives. Medical Journal of Armed Forces India, 68, 304-309. https://doi.org/10.1016/j.mjafi.2012.04.010

[11] Lohani, B. (2013) Pair for Hepatic Hydatid Cyst: A Study in Tertiary Care Hospital in Nepal. Journal of Institute of Medicine, 35, 17-23.

[12] EI Sorogy, M., EI-Hemaly, M. and Aboelenen, A. (2015) Pancreatic Body Hydatid Cyst: A Case Report. International Journal of Surgery, 6, 68-70. https://doi.org/10.1016/j.ijscr.2014.11.062

[13] Stojkovic, M., Rosenberger, K., Kauczor, H.-U., Junghanss, T. and Hosch, W. (2012) Diagnosing and Staging of Cystic Echinococcosis: How Do CT and MRI Perform in Comparision to Ultrasound? PLoS Neglected Tropical Diseases, 6, e1880. https://doi.org/10.1371/journal.pntd.0001880

[14] Eris, C., Akbulut, S., Yildiz, M.K., Abuoglu, H., Odabasi, M., Ozkan, E., Ayalay, S. and Gunay, E. (2013) Surgical Approach to Splenic Hydatid Cyst: Single Center Experience. International Surgery, 98, 346-353.

https://doi.org/10.9738/INTSURG-D-13-00138.1

[15] Llica, A.T., Kocaoglu, M., Zeybek, N. and Guven, S. (2007) Extrahepatic Abdominal Hydatid Disease Caused by Echinococcus granulosus. Imaging Features. American Journal of Roentgenology, 189, 337-343.

[16] Abdelraof, A., Abd EI-Aal, A.A., Shoeib, E.Y., Attia, S.S., Hanafy, N.A., Hassani, M. and Shoman, S. (2015) Clinical and Serological Outcomes in Different Surgical Approches for Hepatic Human Hydatidosis. Journal of Bacteriology and Parasitology, 6, 231.

[17] WHO Informal Working Group (2003) International Classification of Ultrasound Images in Cystic Echinococcosis for Application in Clinical and Field Epidemiological Settings. Acta Tropica, 85, 253-261. https://doi.org/10.1016/S0001-706X(02)00223-1

[18] Dervenis, C., Delis, S., Avgerinos, C., Madariaga, J. and Milicevic, M. (2005) Changing Concepts in the Management of Liver Hydatid Disease. Journal of Gastrointestinal Surgery, 9, 869-877.

[19] Rexiati, M., Mutalifu, A., Azhati, B., Wang, W., Yang, H., Sheyhedin, I. and Wang, Y. (2014) Diagnosis and Surgical Treatment of Renal Hydatid Disease: A Retrospective Analysis of 30 Cases. PLoS ONE, 9, e96602. https://doi.org/10.1371/journal.pone.0096602

[20] Ahmed, Z., Chhabra, S., Massey, A., Vij, V., Yadav, R., Bugalia, R., Kankaria, J. and 
Jenaw, R.K. (2016) Primary Hydatid Cyst of Pancreas: Case Report and Review of Literature. International journal of Surgery Case Report, 27, 74-77. https://doi.org/10.1016/j.ijscr.2016.07.054

[21] Siracusano, A., Teggi, A. and Ortona, E. (2009) Human Cystic Echinococcosis: Old Problems and New Perspectives. Interdisciplinary Perspectives on Infectious Diseases, 2009, Article ID: 474368.

[22] Nabi, M.S. and Waseem, T. (2010) Pulmonary Hydatid Disease: What Is Optimal Surgical Strategy? International Journal of Surgery, 8, 612-616.

[23] Sengul, A.T., Buyukarabacak, Y.B., Yetim, T.D., Gurz, S., Demirag, M.K. and Basoglu, A. (2013) Treatment Strategy for Hydatid Cysts with Multiorgan Involvement/Localization. Journal of Experimental and Clinical Medicine, 30, 5-9. https://doi.org/10.5835/jecm.omu.30.01.002

[24] Rajesh, R., Dalip, D.S., Anupam, J. and Jaisiram, A. (2013) Effectiveness of Puncture-Aspiration-Injection-Reaspiration in the Treatment of Hepatic Hydatid Cysts. Iranian Journal of Radiology, 10, 68-73. https://doi.org/10.5812/iranjradiol.7370

[25] Gupta, N., Javed, A., Puri, S., Jian, S., Singh, S. and Agarwal, A.K. (2011) Hepatic Hydatid: Pair, Drain or Resect. Journal of Gastrointestinal Surgery, 15, 1829-1836. https://doi.org/10.1007/s11605-011-1649-9

[26] Smego, R.A., Bhatti, S., Khaliq, A.A. and Beg, M.A. (2003) Percutaneous Aspiration-Injection-Reaspiration Drainage plus Albendazole or Mebendazole for Hepatic Cystic Echinococcosis: A Meta-Analysis. Clinical Infectious Diseases, 37, 1073-1083.

[27] Blanton, R.E., Wachira, T.M., Zeyhle, E.E., Njoroge, E.M., Magambo, J.K. and Schantz, P.M. (1998) Oxfendazole Treatment for Cystic Hydatid Disease in Naturally Infected Animals. Antimicrob Agents Chemotherapy, 42, 601-605.

[28] Valizadeh, M., Haghpanah, B., Badirzadeh, A., Roointan, E., Fallahi, S. and Raeghi, S. (2017) Immunization of Sheep against Echinococcus granulosus with Protoscolex Tegumental Surface Antigens. Veterinary World, 10, 854-858. https://doi.org/10.14202/vetworld.2017.854-858

[29] Health, D.D. and Koolaard, J. (2012) Serological Monitoring of Protection of Sheep against Echinococcus granulosus Induced by the EG95 Vaccine. Parasite Immunology, 34, 40-44. https://doi.org/10.1111/j.1365-3024.2011.01341.x 